\title{
Applicability of the PIV system for velocity field measurement inside the ultrasonic flowmeter
}

\author{
Jan Novosád ${ }^{1 *}$, Jaroslav Pulec ${ }^{1}$, and Petra Dančová ${ }^{1}$ \\ ${ }^{1}$ Technical University of Liberec, Studentská 2, Liberec, Czech Republic
}

\begin{abstract}
Ultrasonic (US) flow meters are devices for a fluid flow measurement using the ultrasonic principle. Current research project dealing with developing the ultrasonic flowmeter with an innovative channel shape. Previously the work was aimed to the topology optimization using the CFD methods. The final prototype has to be analyzed experimentally to verify the velocity profiles in the US measurement section. The obtained velocity profiles in this region are necessary as the input for the US sensor data evaluation. A particle image velocimetry (PIV) was selected as a potentially suitable technique for the velocity profiles measurement. This paper presents the development and initial testing of a new experimental setup including the experimental track and PIV system. Several modifications were realized during the preparation of the experimental setup. Finally, measurements were performed with a simplified model and the results were evaluated in the form of velocity profiles in the area of interest. The most important results are the knowledge about the requirements on the model preparation. The future work will be aimed to the prototype of the ultrasonic flowmeter.
\end{abstract}

\section{Ultrasonic flowmeter development}

Ultrasonic (US) flow meters are devices for a fluid flow measurement using the ultrasonic principle. The flow rate is related to the fluid velocity. When the pressure disturbances propagate at the speed of sound, and the fluid has also momentum, then the absolute velocity of the pressure disturbance propagation is the algebraic sum. This principle works in several ways as an operating principle of an ultrasonic flow meter. The term ultrasonic means that the pressure disturbances are short bursts of sine waves at frequencies above $20 \mathrm{kHz}$, typically.

The newly designed flowmeters have to be tested regarding the requirements of OIML R137 [1]. This recommendation applies to gas meters intended to measure quantities of gaseous fuels or other gases. The most important test is the flow disturbance test. Flow disturbance tests shall be executed using the applicable piping configurations presented in the OIML R137, mounted upstream of the meter.

Our team works on the research project dealing with developing the ultrasonic flowmeter with an innovative channel shape. A previously published article [2] described the virtual flow disturbance test development using the CFD (Computational Fluid Dynamics) methods.

\footnotetext{
* Corresponding author: jan.novosad@tul.cz
} 
Figure 1 illustrates the 3D CAD model of the innovative design US flowmeter DN80 fluid volume.

The flowmeter assembly is based on the two straight pipes connected together and with the input/output with several elbow segments. This composition leads to the phenomenas described by Idelchik [3] as the variation of the stream direction in curved conduits. The passage of a stream from the straight pipe to the curved section is accompanied by an increase/decrease of the pressure at the outer/inner wall. Then the diffuser phenomena lead to a separation of the stream as illustrated in Figure 2.

The previous study [2] aimed for the CFD analysis of the fluid flow field inside the ultrasonic gas meter. The numerical analysis for the sensibility of the disturbance mounted upstream of the gas was carried out. The study investigated velocity profiles in the ultrasonic measurement zone. The profiles evaluation aimed to confirm that the velocity field (profile) is flow independent. Furthermore, it was necessary to prove insensitivity to input disturbance. Based on the results, the profiles can be considered invariant when the flow rate changes. Comparison of the straight pipe and the disturbance piping mounted upstream of the gas meter indicates that the disturbance does not affect the velocity field in the measurement area. In general, the analyzed design seems to be suitable for stabilizing the velocity field for ultrasonic measurements. Finally, the research continues with some design modifications required by the reduction of the flowmeter casing. The final prototype has to be analyzed experimentally to verify the velocity profiles in the US section. The real velocity profiles are necessary as the input for the US sensor data evaluation.

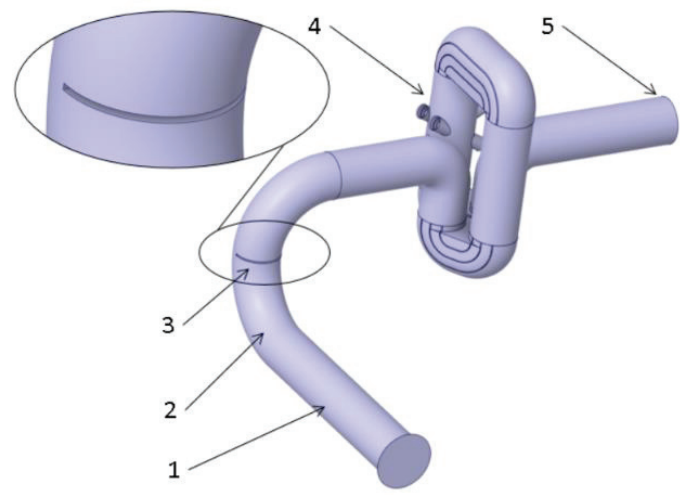

Fig. 1. US flowmeter fluid volume - 3D CAD model with the double bend disturbance [2]: 1 - inlet pipe, 2 - double bend, 3 - half-moon plate, 4 - measuring section with ultrasonic sensors, 5 - outlet pipe.

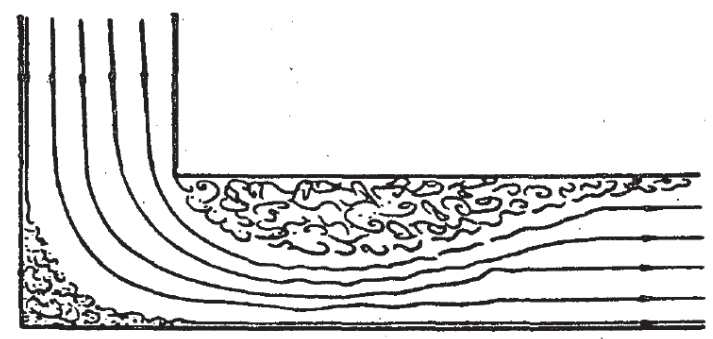

Fig. 2. Stream pattern in a $90^{\circ}$ elbow [3] 


\section{Particle image velocimetry}

In the field of fluid flow measurement, particle image velocimetry (PIV) is an emerging technique. It has greater reliability and convenience, particularly for micro-sized applications than other conventional methodologies that have been applied traditionally in this field of research, such as hot-wire anemometer and Pitot tube that require probes to be present inside the flow to measure. On the other hand, PIV is a non-intrusive measurement technique, which applies laser and light techniques to find the trace of the flow of particles inside the tubes or ducts. Therefore, in this technique, the flow is not likely to be impacted or deformed, enabling the increased possibility for accurate measurements. [4]

PIV is a flow diagnostic technique based on the imaging of the fluid flow, including the seeding particles. The basic underlying image processing technology is the tracking of particles or groups of particles [5]. The basic principle is to record the displacement of illuminated microparticles perfectly dispersed in the fluid flow by a digital image sensor and its subsequent digital evaluation. Laser light is illuminating the targeted region. A system of cylindrical lenses forms the laser beam into the light plane (2D and stereoscopic PIV) or into the light volume (tomographic PIV). The particles are illuminated by two laser pulses with a clearly defined time interval, and their image is recorded with photographic film or digital cameras at two different time points in a specified time interval. A well-developed computerintensive PIV image processing procedure is employed to determine the displacements pertaining to each tracer particle or, usually, groups of tracer particles. The resulting images are then evaluated using the basic equation $v=\Delta s / \Delta t$, where $v\left(\mathrm{~m} \cdot \mathrm{s}^{-1}\right)$ is the velocity of the particle, $\Delta s(\mathrm{~m})$ is its displacement between two images, and $\Delta t(\mathrm{~s})$ is the time step between images (laser pulses). It is assumed that the tracer particles move with identical velocity as local working fluids when deducing the working fluid's velocity PIV techniques for the velocity measurement. The principle of the PIV method is illustrated in Figure 3. Detailed descriptions about the seeding particles were presented by Melling [7]. Additionally, Westerweel [8] described the fundamentals of digital PIV aimed at the mathematical apparatus for the particles velocity evaluation.

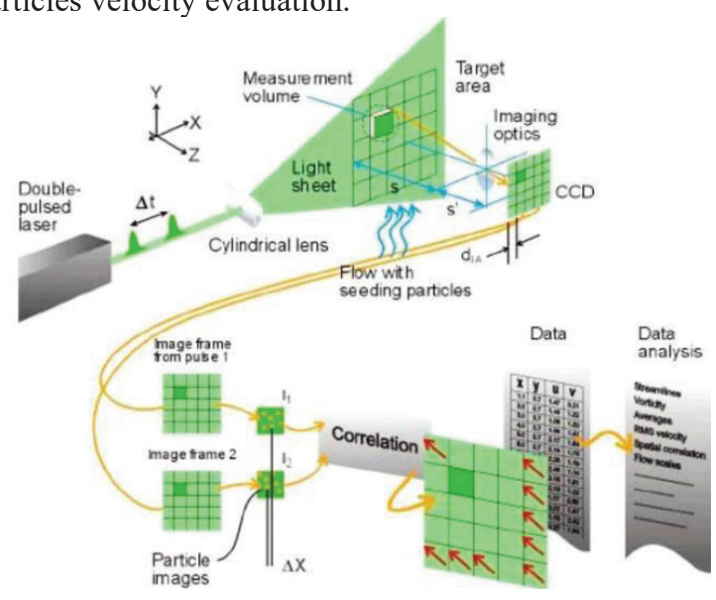

Fig. 3. Experimental arrangement and image acquisition for digital PIV [6]. 


\section{Experimental setup}

The PIV system in the laboratory at the Department of Power Engineering Equipment at the Technical University of Liberec consists of several main components. The whole system is manufactured by the LaVision company [9] with the following specification:

- FlowMaster system from LaVision for 2D, stereo-PIV, and tomo-PIV.

- 4 pcs sCMOS 5.5 MPx camera, resolution $2560 \times 2160$ pixels, 16 bit, a minimum time interval of two frames $120 \mathrm{~ns}$, exposure time $15 \mu$ s to $100 \mathrm{~ms}$, spectral range $370 \mathrm{~nm}$ to $1100 \mathrm{~nm}$, maximum frame rate $50 \mathrm{~Hz}$.

- Nd: YAG double-cavity laser, $200 \mathrm{~mJ}$ per pulse, wavelength $532 \mathrm{~nm}$, maximum repetition frequency $15 \mathrm{~Hz}$, pulse duration up to $10 \mathrm{~nm}$, class 4 laser.

- $1 \mathrm{PC}$ for recording and evaluation, one independent PC for evaluation, Windows 10, DaVis 10.0 software.

\subsection{Measurement of velocity field inside flowmeter model}

The flow field measurement inside the US flowmeter, especially in the region around the ultrasonic probes called measuring area, is the primary goal of our research. The design of the experiment is illustrated in Figure 3. The target area is composed of a transparent pipe with dimensions corresponded to the US flowmeter DN80. In this section, the flow field will be investigated by the PIV method, so the two-section planes for laser sheets are defined (see Figure 3a).

Firstly, the PIV system should be tested and verified for the possibility of the measurement inside and for finding the requirements on the experimental model design and preparation. The simplified physical model was designed and prepared, as shown in Figure $3 \mathrm{~b}$. The main goal of the measurement with this simplified model is to verify the ability of the used materials and experimental setup for PIV measurement in this type of task. Accordingly to the real US flow meter, two vertical pipes DN80 are mounted between the bends with $R / D=1$. Pipes are made of $5 \mathrm{~mm}$ thick plexiglass. The rest of the model components are made of sheet metal with $2 \mathrm{~mm}$ thickness. As illustrated in Figure 3B, the air is sucked from the surroundings into the straight pipe, flows through a first transparent pipe, labeled as "Pipe 1". Then, two elbows lead the air into the second transparent pipe, labeled as "Pipe 2". For the benchmarking case, the experiment is designed as a 2D PIV setup. For this case, one camera located orthogonally to the laser sheet is used for image recording. The laser sheet is aligned with the axis of transparent pipes.

The fluid flow through the pipe elements and devices is significantly influenced by the flow rate. That's why several flow rates were chosen as the operating conditions for the experiment. The flow control is performed by the suction fan at the end of the pipe track. Table 1 notes the flow rates at the inlet section and corresponding velocities and Reynolds number, which is calculated for diameter $\mathrm{d}=80 \mathrm{~mm}$ and the viscosity of air $1.56 \cdot 10^{-5} \mathrm{~m}^{2} / \mathrm{s}$ at $25^{\circ}[10]$. 
a)

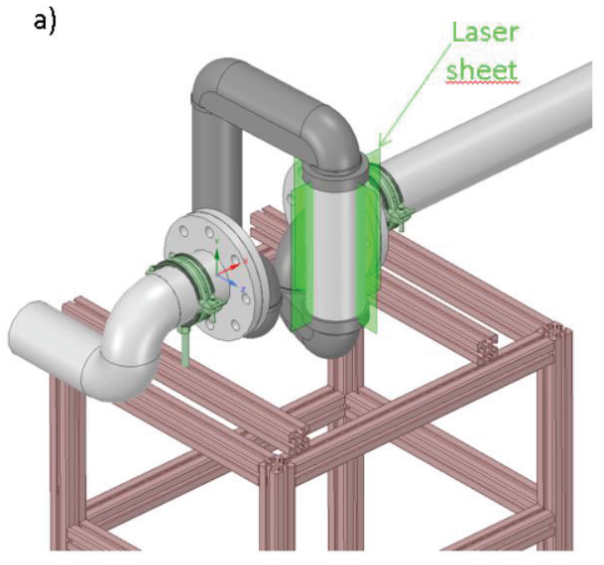

b)

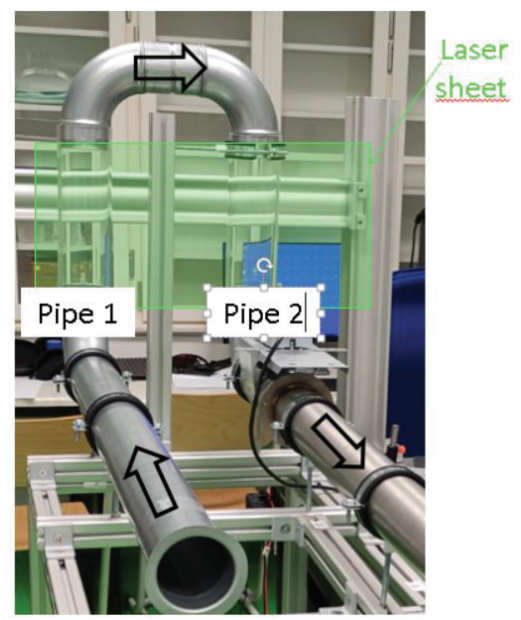

Fig. 4. Experimental setup: a) Design of the experiment for the US flowmeter, b) simplified model for PIV system verification

Table 1. Experimental setup - operating conditions (4 variants).

\begin{tabular}{|c|c|c|c|c|}
\hline Quantity & V01 & V02 & V03 & V04 \\
\hline Flow rate $\left(\mathrm{m}^{3} / \mathrm{h}\right)$ & 8 & 26 & 34 & 69 \\
\hline Mean velocity $(\mathrm{m} / \mathrm{s})$ & 0.44 & 1.44 & 1.88 & 3.82 \\
\hline Reynolds number $(1)$ & 2254 & 7375 & 9629 & 19565 \\
\hline
\end{tabular}

The preparation of the system and initial measurement shows several problems. The most difficult case is the reflection and of laser light inside the rounded plexiglas pipe wall. The troubleshooting is the black cover of a part of the pipe wall (see Target areas in Figure 4). Then the $532 \mathrm{~nm}$ filter is mounted on the camera lens to avoid the environmental light. Additionally, the adjustable aperture with a narrow gap was installed between the camera and target area to limit the recorded area out of the most significant reflection zones. Available calibration plates are not suitable for this task because of the dimensions. The self-printed calibration target was used inside the pipes.

The laser intensity was tuned, and the level at $14 \%$ of the maximum power was finally used. The time between the two images was $330 \mu$ s, and the exposures time was set up to 15 $\mu$ s. The frequency of the laser pulse was $15 \mathrm{~Hz}$. Totally 300 double images were captured for each flow rate. 


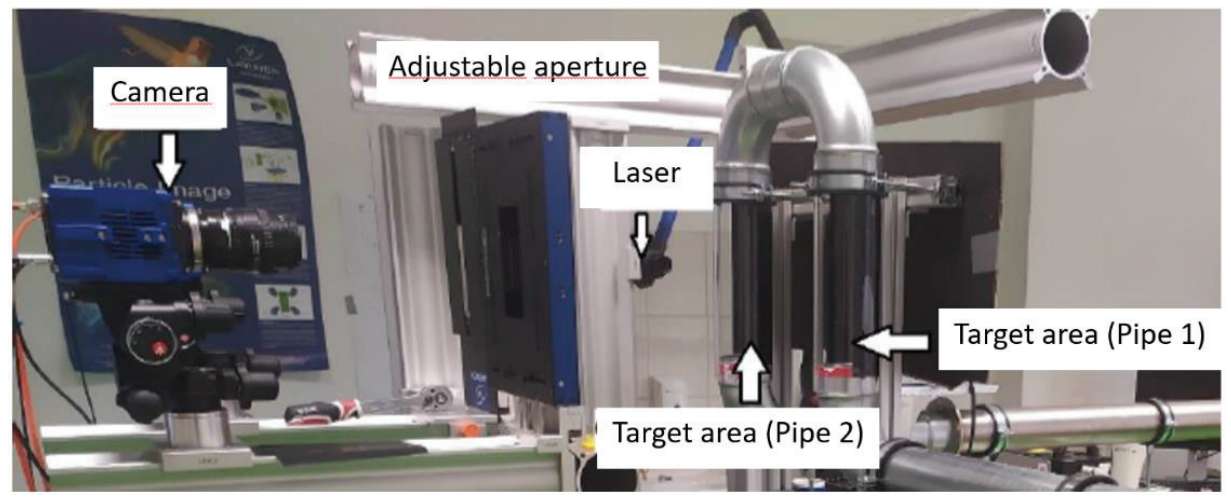

Fig. 5. Experimental setup with troubleshooting (black foil in pipes and adjustable aperture).

\section{Results}

The velocity fields are evaluated in the DaVis software. The postprocessing of the captured images was tuned to reach good results. The main parts of the process were the particle image velocimetry, including the image preparation, vector calculation, and finally, wrong vectors filtering. The results are presented as the selected color maps for target area in pipe 2 are shown in Figure 6.
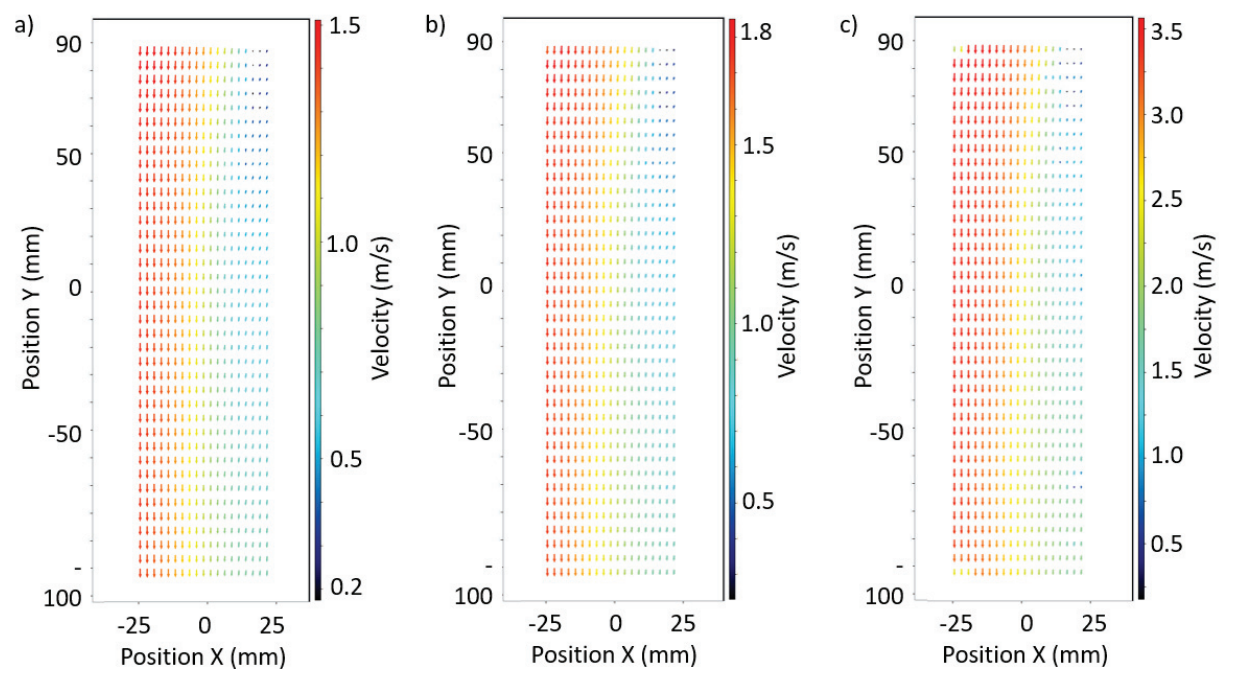

Fig. 6. Experimental results (colormap) - velocity fields, pipe 2 .

The velocity profiles were evaluated from the experimental data at vertical position $Y=\{-$ $40,0,40$ and 80$\} \mathrm{mm}$. Dimensionless position $X(-1,1)$ corresponds to the range $(-25,25)$ $\mathrm{mm}$. The areas close to the wall was not recorded because of the reflection reduction. The dimensionless velocity $U^{\prime}(1)$ is given as $U / U_{\max }$, where $U_{\max }(\mathrm{m} / \mathrm{s})$ is the maximum velocity for each variant. The results for pipe 1 and pipe 2 are shown in Figure 7 and Figure 8. The presented curves show the dependency of velocity on the flow rate. Then, the area 
with low velocities (a wake upstream the elbow) is decreased with higher flow rates, i.e. the flow is better distributed inside the straight pipe.

Comparison of results shows higher maximum velocity values in pipe 2 which is caused by the wakes after elbows. This corresponds to larger effective flow cross section in pipe 1 . For the measurement the seeding of particles in the first pipe is worse, because of less mixing of the air in the previous section. It leads to some error points in the profile which weren't filtered by the automatic postprocessing tools.

a)

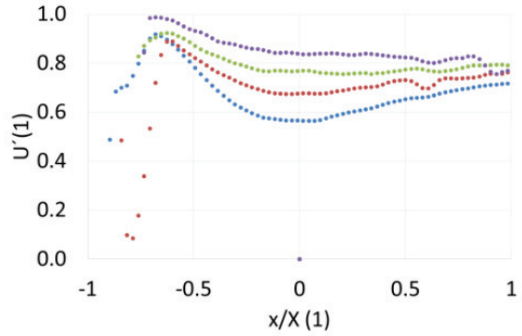

c)

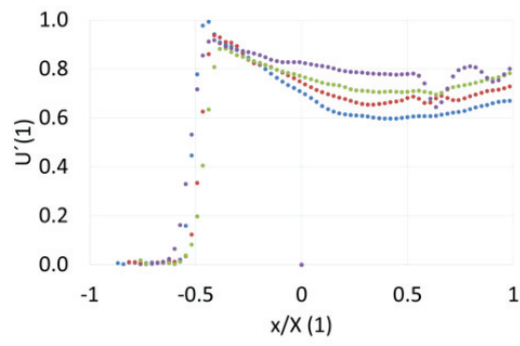

b)

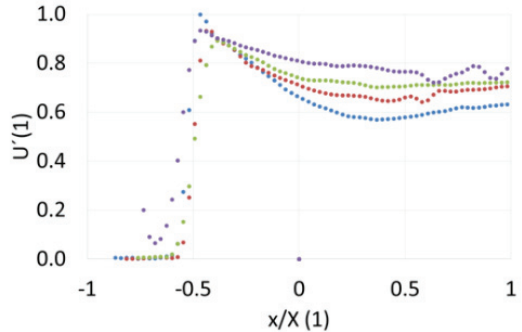

d)

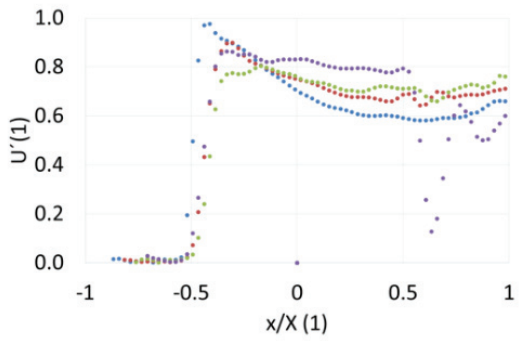

$\cdot Y=-40 \mathrm{~mm} \cdot \mathrm{Y}=0 \mathrm{~mm} \cdot \mathrm{Y}=-40 \mathrm{~mm} \cdot \mathrm{Y}=80 \mathrm{~mm}$

Fig. 7. Velocity profiles for pipe 1 . $U_{\max }$ a) $0.31 \mathrm{~m} / \mathrm{s}$, b) $1.16 \mathrm{~m} / \mathrm{s}$, c) $1.45 \mathrm{~m} / \mathrm{s}$, d) $2.76 \mathrm{~m} / \mathrm{s}$.

a)

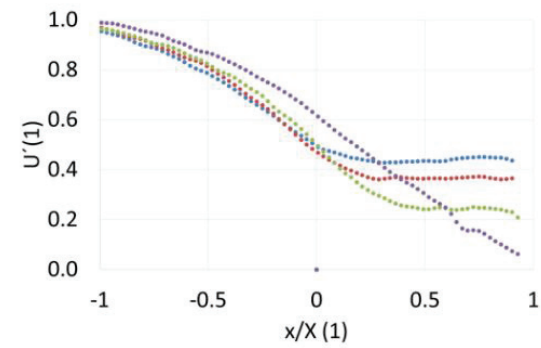

c)

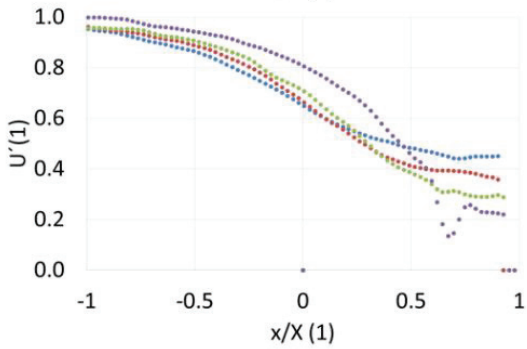

b)

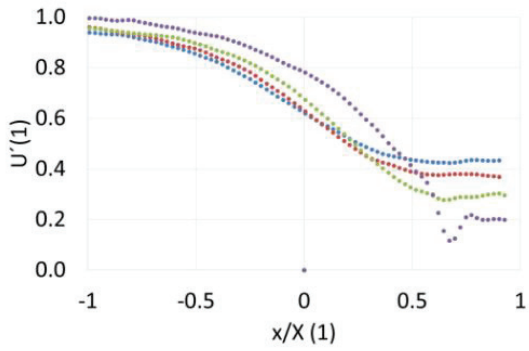

d)

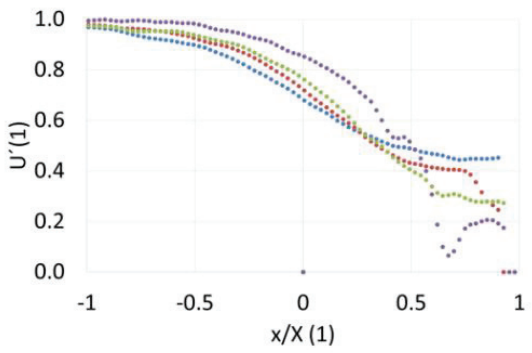

$Y=-40 \mathrm{~mm} \cdot Y=0 \mathrm{~mm} \cdot Y=-40 \mathrm{~mm} \cdot Y=80 \mathrm{~mm}$

Fig. 8. Velocity profiles for pipe 2. $U_{\max }$ a) $0.41 \mathrm{~m} / \mathrm{s}$, b) $1.53 \mathrm{~m} / \mathrm{s}$, c) $1.86 \mathrm{~m} / \mathrm{s}$, d) $3.58 \mathrm{~m} / \mathrm{s}$. 


\section{Conclusion}

In this paper, the experimental work on the PIV system was presented. The simplified model of the newly developed ultrasonic flowmeter was made and tested with the PIV measurement. During the preparation of the experimental setup, several important requirements have been identified. The reflection and refraction caused by the cylindrical transparent model have to be avoided by installing the black foils, camera filters, and adjustable apertures. This leads to the limitations of the measurement, especially for the limit of evaluated data. The results do not describe the areas close to the walls of the target region. On the other hand, the results show the dependency of the flow field inside the channel on the flow rate. The obtained velocity profiles will be useful in the ultrasonic flowmeter development to verify the numerical simulation of the flow field inside the US flow meter.

For future work, some changes are recommended. The experimental setup and methodology of results assessment will be used for the flowmeter prototype. The inner channel shape of the prototype will be more complicated, so the manufacturing of the model should be based on a special technology for making transparent parts. The use of silicone or resin is expected. Choose of material should respect the optical properties to decrease reflections. Additionally, the seeding of particles could be improved to be more homogeneous at the inlet, which should lead to fewer errors in the pipe one region.

This publication was written at the Technical University of Liberec, Faculty of Mechanical Engineering, with financial support from the Czech Technological Agency under the project TACR FW02020048. Part of this work was realized as a part of the project "SGS-2019-5073" with the support of the Specific University Research Grant, as provided by the Ministry of Education, Youth and Sports of the Czech Republic in the year 2021.

\section{References}

1. International organization of legal metrology. Gas meters. OIML R 137-1 \& 2 Edition 2012.

2. T. Vít, J. Novosád, P. Dančová. Int J. Eng Tech \& Inf. (2021);2(1):01-03. DOI: 10.51626/ijeti.2021.02.00003.

3. I. E. Idelchik. Handbook of hydraulic resistance. Research Institute for Gas Purification, Moscow, Russia (1960).

4. M. R. Abdulwahab et al. Journal of Advanced Research in Fluid Mechanics and Thermal Sciences, Volume 65, Issue 2 (2020) pp. 213-229.

5. Wieneke, B. PIV Uncertainty Quantification and Beyond. (2017). DOI:10.4233/uuid:4ca8c0b8-0835-47c3-8523-12fc356768f3.

6. M. Jahanmiri. Particle image velocimetry: Fundamentals and its applications. Chalmers University of Technology, 2011.

7. Melling, A. (1997). Tracer particles and seeding for particle image velocimetry. Measurement Science and Technology, 8, pp. 1406-1416.

8. J. Westerweel. Meas. Sci. Technol. 8 (1997) pp. 1379-1392.

9. LaVision GmBH. www.lavision.de.

10. K. Kadoy, N. Matsunaga and A. Nagashima. J. Phys. Chem. Ref. Data, Vol. 14, No. 4 (1985) pp. 947-970. 